\title{
REVIEWING THE THERAPEUTIC COMBINATION OF MULTIVITAMINS
}

\section{Bharat Kwatra, Chelsea Rumao, Sherin Layanal, Vaishnav Pillai}

Invenzion Labs Inc, Sophia College (Autonomous)- Mumbai, Rajalakshmi Engineering College- Chennai, Vellore Institute of Technology- Chennai.

Article Info: Received 02 August 2021; Accepted 13 September 2021

DOI: https://doi.org/10.32553/ijmbs.v5i9.2218

Corresponding author: Bharat Kwatra

Conflict of interest: No conflict of interest.

\begin{abstract}
Vitamins are micronutrients which form an essential part of our diet. They are needed for healthy functioning of metabolic activities, some forms of vitamin form basis for our immunity and bone building. Usually essential nutrients cannot be synthesised in our bodies, hence we need to obtain them from our diet. Many Vitamin groups have been studied extensively for the pharmacological effects. Many studies have proved the effectiveness of combination therapies of vitamins with other medication for treatment of various diseases. This review presents all the studies conducted to prove the therapeutic effects of vitamins.
\end{abstract}

Keywords: Multivitamins, Vitamin A, Vitamin B, Vitamin C, Vitamin D, Ginseng, Niacinamide, Antibiotics, Cefuroxime, DAV Therapy.

\section{Introduction}

Consuming nutrient-rich diets are better off than others, but care must be taken to check daily consumption of food and any other medication taken for optimal health of the child or adolescent. Nutritional frauds have been on the rise for quite a few years. Duping by misrepresentation of products by catchy marketing and brand promotions as well as lack of proper monitoring and communication among the people in the thick of things have gone a long way to contribute to this phenomenon. Linus Pauling, Nobel Laureate was a proponent of heavy Vitamin $\mathrm{C}$ dosage. But, the human body can't absorb more than 200-250 mg dosage of vitamins, Herbert has warned. But, Dr. Pauling persisted with a $3000 \mathrm{mg}$ vitamin dosage recommendation for his followers. The situation of these nutritional fads have worsened with time with the heavy celebrity endorsements and high budget advertisements causing misconceptions among the general public and these will cause great harm if the masses don't contemplate their stance on the issue. Because of this, a lot of us lack the essential nutrients needed for the healthy functioning of the body.

Vitamins have been studied extensively for its therapeutic effects when given in combination with other medications. Along with Vitamin A, ginseng is also studied for its pharmacological applications. The structural features of ginseng, the pathogenicity of microbial infections, and the immunomodulatory, antiviral and antibacterial effects of ginseng are discussed in this paper. Following the similar context, the pharmacological effects of Vitamin A in cancer and lung infections along with its safety is reviewed. Vitamin $B$ family including Nicotinamide, biotin etc in treatment of sepsis and toad skin disease is discussed. The Vitamin $\mathrm{C}$ and $\mathrm{D}$ families have also been studied upon to know the therapeutic effects of them in many common diseases.

\section{Methods:}

The study was conducted using four databases: Google Scholars, SAGE, DOAJ, and PubMed. The selection of papers was done based on keywords and themes relevant to this review. Further, the published papers from these databases were arranged in systematic order with respect to the year of publication.

\section{Results And Discussion:}

Therapeutic Effects of Vitamin A. 
Vitamin A is an essential nutrient required for vision, reproduction, the integrity of membrane structures, normal functioning of body cells, growth and development. Current nutritional recommendations for dietary vitamin A are well supported by biological evidence that consuming retinol at recommended levels is both safe and sufficient to support vision and maintain systemic functions. Exploratory studies of vitamin A intake is inherently complicated because vitamin $\mathrm{A}$ is consumed partly as carotenoids, often grouped with other antioxidant vitamins, and partly as preformed retinol in food of animal origin and supplements. Frequency questionnaires are imperfect tools for capturing vitamin A intake. Most of them are only qualitative or semiquantitative, and therefore derived data on vitamin A consumption is likely to be weak.

\section{Vitamin in treatment of Cancer.}

Retinoids are one of the most effective classes of agents in promoting cell differentiation and therefore are of great interest in cancer prevention and cancer treatment. The predominance of data supported the reduction of cancer risk when consuming a diet rich in fruits and vegetables, and therefore carotene, but there was little evidence for a relationship with dietary retinol. The association of cancer risk with serum retinol was also weak. A weak relationship can be expected because serum retinol concentrations are maintained at a relatively constant level in a variety of vitamin A states. In addition, if lower retinol levels are measured after disease progression, this may be due to cancer rather than a predictor. Recent reports from epidemiological studies on the prevention of prostate and breast cancer have concluded that higher dietary intake of fruits and vegetables and vitamin A has not been associated with the prevention of these cancers. Overall, therefore, the epidemiological evidence for reducing the risk of cancer by consuming foods containing provitamin A carotenoids or preformed vitamin A is relatively weak. Retinoid intervention studies for cancer chemoprevention or therapy have been conducted for more than three decades. In another study of current and former smokers, $50,000 \mathrm{IU} / \mathrm{d}$ retinol for 6 months did not regulate
RAR- $\beta$, which, as noted above, is often silenced in cancer or improved surrogate biomarkers of bronchial dysplasia. One type of cancer excels with remarkable success.

Due to its benefits for supporting vision, a person may choose to consume more of their vitamin A intake in the form of $\beta$-carotene, rather than preformed retinol, since consuming $\beta$-carotene at nutritional levels poses no risks to toxic organisms. Based on the cancer literature reviewed in this chapter, there is no convincing evidence that increasing dietary vitamin A intake above current recommended levels will reduce the risk of developing cancer.

\section{Vitamin A in treatment of Lung Infection and Cystic Fibrosis}

In CF lung disease, there is a chronic bacterial infection and the resulting inflammation controlled by neutrophils has led to a massive release of proteases and reactive oxygen species into the airways. Most patients with cystic fibrosis suffer from exocrine pancreatic insufficiency with indigestion and malabsorption of fats and proteins. Pancreatic enzyme replacement therapy may prevent steatorrhea but may not normalize the absorption of fat-soluble vitamins. Vitamin A is supplemented in all CF patients with PI, but the recommended doses are between centres. Animal studies have also shown that vitamin A supplementation can increase anti-inflammatory activity. Fitzgerald has demonstrated the effects of several factors on vitamin A absorption in patients with cystic fibrosis. The Fitzgerald procedure determines normal or abnormal absorption of vitamins based on an increase in delta above fasting serum levels. In one of the studies conducted, it was found that serum vitamin A levels correlated with lung function. The use of the 5th percentile to define hypovitaminosis A was dictated by statistical, not biological reasoning, and we cannot argue that this value is the optimal cut-off for determining low SRL in patients with cystic fibrosis. In another small study of 38 patients with cystic fibrosis, all of whom were in clinically stable condition, a significant correlation between SRL and ppFEV1 was observed. The results showed an association between vitamin $\mathrm{A}$ 
and lung function in cystic fibrosis, but unfortunately, this could not prove a cause-andeffect relationship and shed light on the nature of the underlying pathophysiology.

\section{Pharmacological Effects of Ginseng}

Ginseng has been used for centuries as a traditional medicine to treat numerous ailments, but little clinical research has been done on its effect on respiratory infections. Most clinical studies have focused on investigating the possible effects of ginseng on fatigue, memory, menopausal symptoms, and mild diabetes symptoms. Between 2002 and 2017, one hundred and thirty-four trials of ginseng were registered, of which $60.4 \%$ were completed and the remaining $23.1 \%$ are actively recruiting participants.

Ginseng is one such herbal medicine known to relieve pro-inflammatory chemokines and cytokines formed by macrophages and epithelial cells. Various clinical trials with ginseng have shown a reduction in repeated colds and flu. the latest animal studies and human clinical trials that support the role of ginseng as a therapy for the treatment of respiratory tract infections. Ginseng products are generally used as complementary and alternative medicine in respiratory infections. The effectiveness of ginseng has been evaluated in numerous clinical trials exploring the treatment of colds and flu. The results showed that ginseng relieves symptoms and prevents respiratory infections. COLD-fX, which is isolated from the roots of American ginseng, is effective and safe against respiratory pathogens, as well as reducing the viral load of patients prone to seasonal influenza. The extract's ability to stimulate the release of IL-2 and IFN could be attributed to its effectiveness against respiratory infections. In addition, COLD-fX reduced the relative risk and duration of respiratory symptoms by $48 \%-55 \%$, in elderly patients who were immunocompromised during influenza season. A dose of $100 \mathrm{mg}$ ginseng twice daily for 12 weeks improved the pulmonary function test of respiratory endurance in 92 patients with COPD. In two groups of patients, the first group received $875 \mathrm{mg}$ of amoxicillin and 125 mg of clavulanic acid, while the second group received antibacterial treatment with $100 \mathrm{mg}$ of standardized G115 ginseng extract twice daily for nine days. Bacterial clearance was significantly faster for the ginseng group compared to the antibiotic-only group. They studied the role of ginseng extract in improving quality of life and relieving symptoms.

Panax ginseng is called "Ren Shen" in China because of its upright humanoid-like shape. "Ren" means people in Chinese, so it can be seen that ginseng is inextricably linked with the human body and plays an important role in regulating human health and treating different diseases. To improve the level of clinical evidence, 25 of the 144 selected studies do not have RCTs and are excluded. Among them, 32 ginseng extract, 34 Korean red ginseng, 22 recipes containing ginseng and 7 ginseng compounds, a total of 95 published articles showed that ginseng has many positive effects, including regulation of blood glucose and metabolic syndrome, reduction of cardiovascular risk factors, and improving cognitive and memory levels, fatigue, physical tolerance, sexual function and quality of life, especially in cancer patients.

Although, combination products containing ginseng as one of several ingredients have been associated with serious side effects and even death. However, fewer side effects were seen in the ginseng-only group, and none of the patients developed 'ginseng abuse syndrome', although the size of the study was small and therefore difficult to interpret.

\section{Safety of Vitamin A}

Vitamin A is an essential nutrient for humans as it cannot be synthesized de novo in the body. Vitamin A adequacy is discussed in terms of recommended allowances appropriate to the needs of the majority of individuals. Globally, reports of vitamin A toxicity are rare and there are very few deaths reported. Acute hypervitaminosis A can result from natural food sources or, more often, from excessive consumption of vitamin A supplements. The most common symptoms include nausea and vomiting, fatigue, headache, swelling of the fontanel, elevated serum vitamin A, and anorexia. Chronic hypervitaminosis A can occur when excessive amounts of vitamin A are 
consumed over long periods. Vitamin A given at very high levels is teratogenic in animals, and a deficiency of the vitamin during pregnancy can also cause birth defects. A few cases of human congenital malformations associated with maternal overuse of high doses of vitamin A have been reported, but no cause-effect relationship has been established. It is recommended that the doses of vitamin A given during pregnancy should not exceed the US RDA value of $2424 \mathrm{RE} / \mathrm{d}$.

\section{The Vitamin B Family}

Vitamin B Family has been critically studied to understand its various properties and its effects on health. Biotin is a vitamin B and has many potential beneficial effects. Biotin is an important substance and its deficiencies cause severe problems. Biotin helps in the improvement of many diseases such as multiple sclerosis. Vitamin B2 has many therapeutic effects and can be used to treat sepsis. The amino acid can supplement the therapy since it promotes muscle repair and other factors. It can complement the vitamin B2 therapeutic effect and help in improved recovery. Vitamin B2 and amino acid is seen to be an effective combination for sepsis therapy, but more investigation is warranted. Many nutrients have been tested in different experiments with variable results for phrynoderma treatment. Vitamin A \& B's intramuscular administrations were found effective against phrynoderma. Nicotinamide is the amide form of vitamin B3. It is an important precursor for substances such as ATP. Nicotinamide use in the treatment of acne vulgaris, melasma, atopic dermatitis, etc. is discussed. Thiamine is also known as vitamin B1. It has the potential to play an important role in nutrition therapy. This article provides a complete understanding of thiamine's role in nutrition therapy and provides various tips for deficiency management. Pyridoxine is also known as vitamin B6. It is suggested that vitamin B6 has many therapeutic effects. It is observed that dietary vitamin B6 has the properties to suppress colon tumorigenesis by decreasing cell proliferation. It is hypothesized that vitamin B12 could reduce the risks of chronic diseases. More research is required to justify that vitamin B12 can protect against chronic diseases. Pantethine is vitamin B5 and can potentially fight against cardiovascular and cerebrovascular morbidity. It can be stated that vitamin B5 is a well-tolerated therapeutic agent with great potential against such severe diseases. Folic acid is vitamin B that holds an essential role in the function of DNA. It is suggested that folic acid could be a potential therapeutic agent. It is observed that folic acid can act as a therapeutic agent in patients with chronic inflammatory skin diseases and other severe cases. Niacinamide is the amide form of vitamin B3, niacin. It is a watersoluble substance. It has many beneficial properties to human health. Niacinamide is a potent therapeutic agent which can be applied in various treatments after further investigation and studies. Vitamin B family together can be a potent option for various therapies. In mammals, Biotin is an essential cofactor for following 5 biotindependent carboxylases: acetyl-CoA carboxylase (ACC) 1, ACC2, methylcrotonyl-CoA carboxylase (MCC), pyruvate carboxylase, and propionyl-CoA carboxylase (PCC). From a previous multivitamin supplementation study, it has been indirectly evident that some degree of biotin deficiency occurring spontaneously during human gestation could be teratogenic. It was also recommended that biotin intake of 2-3 times more than adequate intake (AI) is required during pregnancy. Marginal biotin deficiency in healthy adults can be indicated using urinary 3hydroxyisovaleric acid (3HIA) and 3hydroxyisovalerylcarnitine (3HIAc) when it is induced experimentally. From previous observations and experiments, it is suggested that lactation increases biotin turnover and loss. This turnover was observed in early pregnancy and it returned to normal by late pregnancy. Previous work reported that the most suitable indicators of marginal biotin deficiency are the amount of holoPCC and holo-MCC in peripheral blood lymphocytes, which can be determined by gel densitometry fluorescent-labeled streptavidin. But this technique is quite technically demanding and requires expertise. Holocarboxylase synthetase (HLCS), an enzyme that catalyzes the binding of biotin with apo-carboxylase proteins to form active holocarboxylase, is reported to affect gene 
expressions. High-Dose Biotin Therapy was found to be a successful treatment for Biotin-thiamineresponsive basal ganglia disease (BTBGD), a genetic disorder that affects the nervous system. High-Dose Biotin has responded clinically for cases of optic neuropathies and leukoencephalopathy. The treatment is also represented as a therapeutic option to slow down progressive multiple sclerosis. The mechanism is still unclear but it is hypothesized that it works through increasing myelin production. As the use of biotin as pharmacologic doses increases for adults and children, it could interfere with clinical diagnostic tests using the streptavidin-biotin technique such as to estimate hormones. It is suggested that biotin therapy be mentioned in a patient's history if that person has undergone one.

Sepsis is a serious risk with characteristics such as hypermetabolism, increased energy usage, and augmented peripheral protein catabolism caused by the inflammatory responses. It also leads to losses of constitutive and structural proteins which results in delayed tissue repair, wound healing, and compromised immune function. It is hypothesized that highly purified Vitamin B could be a viable treatment. The treatment could be enhanced with supplementation of amino acids which might reduce the organ damage. Male ICR mice were used for the experiments. They were given an i.v. bolus injection of lipopolysaccharide and after 6 hours, they were given the treatment. There were three experiments: one with Vitamin B2 in saline or aminolevane solution, the second consisted of Vitamin B2 combined with individual amino acids of amino levine solution and the third observed Vitamin B2 with or without valine. To observe the effect of Vitamin B2 on the macrophage function, the lipopolysaccharide-stimulated macrophages were extracted from killed mice and were evaluated for the effects of vitamin B2 on interleukin-6 and lactic acid production, and glucose consumption. There were no survivors after 7 days in the saline and aminolevane solution group. Still, the aminolevane group showed more Vitamin B2 therapeutic effect. Vitamin B2 combined with tryptophan, isoleucine, proline, threonine, alanine, and valine through i.v. treatment showed a significant therapeutic effect.
But only valine showed a significant effect when combined with vitamin B2 in i.v. bolus administration. Vitamin B2 reduced the lipopolysaccharide-stimulated production of interleukin-6. It also inhibited the elevated lactic acid production and improved the excessive consumption of glucose. Aminolevane significantly increased the vitamin B2 therapeutic effect on endotoxin-induced shock, even though it showed no enhancement in lipopolysaccharideinduced mortality. aline could also inhibit the lipopolysaccharide-induced mouse macrophage production of interleukin-6 and TNF-alpha. Vitamin B2 with human activated protein $\mathrm{C}$ treatment also showed a decrease in mortality of toxin-induced shock. vitamin B2 has been shown to normalize the decreased mitochondrial membrane potential of mouse neutrophils exposed to lipopolysaccharide and also blocked the production of excess cytokine from macrophages exposed to mitochondrial electron transporter inhibitors.

Phrynoderma (toad skin) is a type of follicular keratosis. It is hypothesized that nutritional deficiency could be the factor behind it like Vitamin A, Vitamin B complex, essential fatty acids (EFA), and Vitamin E. The observation was graded based on the percentage of flattening and reduction in the number of lesions. A good number of patients showed satisfactory results under regimens 1 and 2 which were Vitamin $A$ and Vitamin B complex respectively. Individual vitamins of the Vitamin B family showed no significant response when used in therapy for the disease. Vitamin B-complex together with EFA as a combination therapy showed better responses than single-drug therapy. A similar observation is noted with combination therapy of Vitamin Bcomplex and Vitamin E. It is concluded that intramuscular injections of Vitamin A or Bcomplex could be effective for the treatment of the said disease. It is also suggested that for better management of the disease a combination therapy of Vitamins and EFA with adequate nutrition be used.

Nicotinamide is a form of Vitamin B3. It is converted from niacin acid in the body. Vitamin 
B3 deficiency results in pellagra. Nicotinamide is considered a food additive and has neuroprotective and antioxidant properties. It also decreases wrinkles, UV-induced immunosuppression, wrinkles, and sebum production. Topical application of nicotinamide has sebo-suppressive, anti-inflammatory, and healing functions and thus is useful to treat acne vulgaris. It also decreases cutaneous pigmentation by limiting the transfer of melanosomes from melanocytes to keratinocytes.It also helps with rosacea and atopic dermatitis as it reduces water loss and increases stratum corneum hydration as well as enhancing the overall structure, elasticity, and moisture of the skin. Oral nicotinamide reduces basal cell carcinomas and actinic keratosis development in patients with nonmelanoma skin cancer. It also showed moderate improvement with acne and rosacea. Excess oral nicotinamide is suspected to be involved in the development of Parkinson's Disease. It is known that nicotinic acid may factor in liver abnormalities. It is also able to cross the human placenta.

Thiamine is converted to thiamine pyrophosphate (TPP) by phosphorylation using the enzyme thiamine pyro(di)phosphokinase. TPP is an important substance as it is involved in several enzyme functions, a cofactor in glycolysis and oxidative decarboxylation, coenzyme for the mitochondrial enzyme complexes $\alpha$-ketoglutarate dehydrogenase, and pyruvate dehydrogenase. It is also important for lactate to pyruvate conversion and enzyme erythrocyte transketolase. Thiamine deficiency can be suspected in patients with a history of alcoholism, AIDS, and malignancies. Other risk factors include pregnancy and lactation, kidney failure, old age, infections, diabetes mellitus, hyperthyroidism, and other serious illnesses. Bariatric surgery and post-bariatric surgery obese patients are also susceptible to thiamine deficiency. To check thiamine, the preferred test is the estimation of thiamine diphosphate in erythrocyte hemolysates using high-performance liquid chromatography. Thiamine is also suggested for recovery from trauma. It can also lead to enhanced wound healing with riboflavin and pyridoxine. Patients with alcohol-related diseases and alcohol withdrawal states are advised to use thiamine replacement in therapy. It is found that Vitamin B6 can work as a chemoprotective agent as it has suppressed the growth of cancer cells in vitro. The article has hypothesized that moderate intake of Vitamin B6 might reduce colorectal cancer risk. It was tested on the development of colon tumorigenesis in azoxymethane (AOM)-treated mice and the results were positive. It was correlated that Vitamin B6's property of lowering cell proliferation leads to its antitumor effect. Pyridoxal 5'-phosphate can act as an inhibitor for enzymes that have binding sites for phosphate-containing substrates such as RNA polymerase, DNA polymerase, etc. It is also reported that vitamin B6 deficiency results in higher lipid peroxidation in the plasma and the liver when the organism consumes a high-fat diet. Vitamin B6 reduced hepatic iNOS activity and lipid peroxide in LPS-treated rats. In angiogenesis, the development of microvessels was inhibited by high levels of pyridoxine and PLP, with PLP being more effective. This implies that further investigation is needed to be done on vitamin B6 and its beneficial effects.

Most of the vitamin B12 deficiencies are due to food-cobalamin malabsorption syndrome caused by gastrointestinal complications. Elevated plasma homocysteine levels are reported as a risk factor for cardiovascular disease. It is suggested that a combination treatment of folic acid and vitamin B12 can lower homocysteine levels. Elevated homocysteine levels could cause atherosclerosis, and administration of an optimum amount of vitamin B12, B6, and folic acid could prevent it and risk of clot formation too. Deficiency of folic acid, vitamin B6, and vitamin B12 are related to cancer through the incorporation of uracil, instead of the appropriate base, into human DNA, which leads to chromosomal breaks. High levels of homocysteine might also be responsible for brain function, apart from heart diseases. Levels of homocysteine are associated with a decline in cognitive performance in a normal aging group. Some studies have shown that supplements of vitamin B12 improve symptoms indicative of delirium or enhance some functions in cognitively impaired patients. Depression is associated with hyperhomocysteinemia, which is vitamin B12 
deficiency, and with impaired 1-carbon metabolism due to genetic polymorphism. It suggests that vitamin B12 supplementation can be used in antidepressant therapies. It is seen that an optimum amount of vitamin B12 might be necessary for folate therapies such as to reduce the occurrence of neural tube defects (NTDs). Food fortification with vitamin B12 will be of great benefit. Vitamin B12 levels optimally influence dementia, depression, as well as the development of cancer concerning genetic polymorphisms.

Pantothenic acid/ Pantetheine is also called vitamin B5. It acts as a precursor of coenzyme A, which is involved in the fat metabolism-related processes, including synthesis, transportation, and degradation of fatty acids. It also plays a role in the breakdown of most amino acids carbon skeletons for the biosynthesis of various biomolecules. Vitamin B6 has a therapeutic effect on serum lipid metabolism, it reduces the de novo synthesis of cholesterol and triglyceride, and promotes lipolysis by increasing the level of coenzyme A in the hepatocytes, and it also regulates the function of hydroxy-methyl-glutaryl-coenzyme A reductase, which again reduces the de novo synthesis of cholesterol. It can be stated that pantethine administration can solve the important problem of protecting the arterial walls from atherogenic effects. Pantethine administration has to lead to a decrease in total cholesterol \& phospholipids in the membrane of the platelets without any change in the proportions. here are three pantethine-promoted platelet antiaggregation mechanisms reported:

1. A sudden anti-aggregating activity after 1 minute was observed, which could be due to the antioxidant property of pantethine.

2. It also decreases the malondialdehyde synthesis of platelets and inhibits aggregation induced by arachidonic acid.

3. Cyclooxygenase-initiated pathway is also limited by pantethine, which could probably be due to membrane fluidity or architecture changes.

Pantethine has endocrine effects such as decreasing prolactin in the pituitary and the plasma and therefore can be used to manage hyperprolactinemia, and it also increases adrenocorticotropic hormone \& cortisol levels. It is also concluded that pantothenic acid promotes the synthesis of acetylcholine in the brain of rodents. It is also to be noted that pantethine may have potential antidepressant properties by enhancing brain-derived neurotrophic factor (BDNF) levels. There are other reported possible effects of pantethine, such as

1. Protection against carbon tetrachloride hepatotoxicity in rats.

2. Reduces negative alcohol effects in chronic alcoholics.

3. Decreases levels of cystine in cystinosis.

4. Inhibition of lens opacification during selenite or other chemicals induced experimental cataract.

5. Prevention of cerebral syndrome in Plasmodium berghei-infected mice, and also the preservation of blood-brain barrier.

Folic acid is a type of water-soluble vitamin B and is important for normal cell growth and metabolism. The recommended daily intake is 0.4 mg. Folate deficiency could be a risk factor for malformations, cardiovascular disease, dementia, and cancer. Supplementation of folate to pregnant women may prevent neural tube defects. Increased intake of folate may decrease breast cancer risk among regular alcohol-consuming women. Folic acid is used as a protection from methotrexate toxicity in dermatology. Folates play important roles in DNA synthesis and epigenetic mechanisms. It is understood that the administration of folic acid might not help with vitiligo. Hyperhomocysteinemia could be a potential factor in the case of deep venous thrombosis (DVT), which can be improved with folic acid and oral anticoagulants administration. Folic acid supplementation can be used as a therapeutic agent to treat psoriasis which has concomitant hyperhomocysteinemia, low plasma folate, and other cardiovascular risks. Folic acid can be a possible supplementation to decrease the toxicity of methotrexate, a compound used to treat psoriasis. Recent studies have reported that it also helps with other congenital abnormalities such as congenital limb deficiencies, pyloric stenosis, 
cardiovascular malformations, and many more. It also reduces inflammatory mediators in atherosclerosis, insulin sensitivity, and other such skin diseases. Further investigation is needed to understand the role of folic acid in other diseases.

Niacinamide is a water-soluble vitamin $B$, converted from nicotinic acid. It is involved in energy metabolism, the regulation of DNA synthesis, and transcription processes. Niacinamide is important for pro-inflammatory mediators and the expression of adhesion molecules. It can also inhibit MHC-II expression, and the production of IL-12, TNF-alpha, IL-1, and NO. It increases vascular permeability and prostaglandin synthesis. This effect can be applied in plastic surgery. The anti-inflammatory effect is mainly due to the inhibition of leukocyte chemotaxis, lymphocyte transformation, and the release of lysosomal enzymes. Niacinamide's barrier-protective effects promote its antipruritic effects. It is also involved in ceramides biosynthesis, which results in the expression of the key enzyme for sphingolipid synthesis i.e. serine palmitoyltransferase. It is a potent cAMPphosphodiesterase inhibitor and stabilizes the mast cells, thus reducing the histamine release. It is reported that niacinamide shows tuberculostatic, antiretroviral, and fungistatic effects. It works efficiently against Mycobacterium tuberculosis and HIV. Niacinamide has a photo-protective property that is based on the inhibition of photocarcinogenesis and the protection from UVinduced immunosuppression. Further studies are needed to apply the beneficial effects of niacinamide into dermatology.

\section{The Vitamin C Family}

Vitamin C(ascorbic acid) has been very effective against infections causing whooping cough, diphtheria, tetanus, polio or infection due to AIDS. Pseudomonas aeruginosa has high resistance to antimicrobials and is "one of the top three causes of opportunistic human infections and a major causative agent of hospital infections in burn patients" Studies dating back to the 1970s indicated "megadoses of ascorbic acid in combination with antimicrobials inhibit Pseudomonas aeruginosa growth". Large doses of
AA work synergistically with suitable antibiotics against acute bacterial diseases. In a study of 12 isolates, 5 clinical ones were obtained from "patients with burns and nurses" and 7 from sewage. Fresh solutions were made of chloramphenicol(Cm),

kanamycin $(\mathrm{Km})$, tetracycline $(\mathrm{Tc})$, streptomycin $(\mathrm{Sm})$, tobramycin( $\mathrm{Tb}$ ) and ampicillin(Ap). Ascorbic acid solution in distilled sterile water was adjusted to $\mathrm{pH} 7.0$ with $10 \mathrm{~N} \mathrm{NaOHand}$ added to the media at appropriate concentration. After incubation $\left(37^{\circ} \mathrm{C}\right)$ for 16-24 hrs, the lowest concentration of antibiotic separately, or in combination with AA, which prevented the development of turbidity was regarded as $\mathrm{MIC}$ (minimum inhibition concentration) ans Fractional Inhibitory Concentration(FIC) was used to interpret the tube dilution method results as, FIC of drug= MIC of drug in combination with AA/ MIC of drug alone. Synergy was detected in 4 antimicrobial-ascorbic acid combinations(those for $\mathrm{Cm}$, Tc, $\mathrm{Km}$ and $\mathrm{Sm}$ ). $\mathrm{Ap}$ and $\mathrm{Tb}$ didn't show synergy with AA. Antagonism was observed for AA-Cm for $8.33 \%$ of the isolates. $\mathrm{Cm}, \mathrm{Km}, \mathrm{Tc}, \mathrm{Sm}$ showed indifference against $41.67-58.3 \%$ isolates. Experiments were conducted on soil samples collected from a dumping ground in South Kolkata. The assay was then carried out using multiple antibiotic-ascorbic acid combinations and antibiotics and ascorbic acid separately. The antibiotics used were Ampicillin(AMP), Linezolid(LZ), Chloramphenicol(CHL) and Azithromycin(AZT) respectively. A slight increase was seen in the zone of inhibition in combination with vitamin $\mathrm{C}$ and $\mathrm{LZ}$ is the most effective antibiotic for treating the sample, alone and in combination with vitamin $\mathrm{C}$.

\section{DAV Therapy}

A novel "synthetic-metabolic" approach is devised with triple combination therapy of Doxycycline, Azithromycin, and Vitamin C (DAV). It is previously shown that mitochondrial biogenesis and oxidative metabolism support CSCs propagation. Doxycycline is a tetracycline-based antibiotic, which acts as an inhibitor of mitochondrial protein translation and thus inhibiting CSCs propagation. The inhibitory action 
of Doxycycline can be enhanced by combining it with Azithromycin and Vitamin C. Experiments showed that a combination of Doxycycline and Azithromycin has better inhibition of mammosphere formation than when used individually. This combination leads to a decrease in respiration and ATP levels as well as in glycolysis and glycolytic reserve, thus suggesting it to be a competent therapeutic option. It was previously observed that Vitamin $\mathrm{C}$ alone inhibits CSC propagation. When combined with Vitamin $\mathrm{C}$, Doxycycline and Azithromycin showed a remarkable increase in inhibition of CSC (by almost 90\%). This combination therapy can be a novel "synthetic-metabolic", feasible anti-cancer approach for targeting CSCs. It is also hypothesized that this triple combination therapy may enhance health and lifespan. Azithromycin is reported to have demonstrated senolytic activity by targeting and removing senescent fibroblasts. Since senescent myofibroblasts are cancerassociated fibroblasts, DAV triple combination may target the glycolytic tumor stroma of cancers.

In conclusion, it can be stated that the DAV triple combination therapy is a viable option to inhibit CSCs but needs further clinical trials to completely validate this as an option for breast cancer patients.

\section{The Vitamin D Family}

Vitamin D is generated from 7dehydrocholesterol, a cholesterol precursor found in the skin. When UV-B radiation reaches the skin, it converts the precursor to previtamin D3, which then undertakes a thermally induced change into vitamin D3 (cholecalciferol). UV radiation is a well-known human carcinogen that is undeniably linked to the current high occurrence of skin cancer. Vitamin D status can only be determined by measuring serum 25-hydroxyvitamin D levels. Vitamin D3 deficiency or insufficiency is known to cause rickets, osteomalacia, and osteoporosis. Vitamin D's roles are not restricted to its significant skeletal effects but are also intimately linked to non-skeletal effects. The number of papers related to "Vitamin D" in the PubMed database has risen over time. In the winter, mainly in the northern states, respiratory tract infections (RTI) are more widespread than in the summer.
This also applies to the pandemic-like spread of the infectious Coronavirus illness 2019 (COVID19) over the world during the winter months, because the virus is more easily spread at low temperatures. Vitamin D is a series of fat-soluble prohormones discovered after the antirachitic activity of cod liver oil was discovered in the early twentieth century. One of medicine's greatest successes must be the discovery of vitamin D and the removal of rickets as a serious medical condition. Sir Edward Mellanby's study was particularly important, as he established that he could cause rickets in dogs by providing them with a Scottish diet, namely oatmeal. Vitamin D insufficiency affects an estimated 1 billion people globally, of all races and ages (VDD). This outbreak of hypovitaminosis D is largely due to environmental and genetic factors that limit sunshine contact. Vitamin D deficiency is common in newborns who are exclusively breastfed and thus do not take vitamin D supplementation, as well as in individuals of all ages, who have darker skin pigmentation, wear sunscreen all the time, or minimize their outside activity.

As the UV action spectra for DNA damage that leads to skin cancer and for vitamin D photosynthesis are almost identical, UV irradiation's detrimental and positive actions are inextricably linked. After the discovery of the antirachitic activity of cod liver oil in the early twentieth century, vitamin D was given its name. The active vitamin 1, 25-2 Vitamin D's major physiologic function is to maintain calcium homeostasis. Vitamin D sustains the calcium 3 phosphate product in a range that gives osteoblasts enough ions to mineralize the collagen matrix by sustaining serum calcium and phosphate levels. Inadequate bone matrix mineralization is caused by a lack of calcium and/or phosphate deposition in the bone matrix. Hypercalcemia and hypercalciuria are the clinical effects of too much vitamin D. As it's easy to measure and has the longest half-life in circulation, the circulating level of the inactive precursor $25-\mathrm{OH}$ vitamin $\mathrm{D}$ is widely considered as the globally accepted indication of vitamin D status. "Sufficiency" traditionally refers to $25-\mathrm{OH}$ vitamin D levels that correspond to the absence of calcium homeostasis 
problems. In the absence of clinical manifestation of osteomalacia or rickets, laboratory values, specifically serum PTH levels within the normal range that can be reduced by vitamin $\mathrm{D}$ supplementation, have been interpreted to indicate an undesirable vitamin D status, even in the absence of disease signs or symptoms. Although evidence of a functional role for vitamin $\mathrm{D}$ in skeletal muscle and the nervous system is confined to the elderly, several studies revealed a decline in fracture risk even when bone mineral density was only slightly enhanced. Vitamin D supplementation reduces the incidence of slips that lead to fractures rather than enhancing bone strength, which is one reason for the decline in osteoporotic fractures. Because melanin in the skin competes with 7-DHC for UVB photon absorption, dark skin pigmentation is linked to a higher risk of vitamin D deficiency, particularly in African Americans. Although both pigmented and non-pigmented skin can produce vitamin D3, pigmented skin needs more exposure durations. In otherwise healthy people, there is no proof that regular sunscreen use induces vitamin D deficiency or insufficiency.

In real-world conditions, sunscreens do not promote vitamin D insufficiency or have an unfavourable effect on bone remodelling and homeostasis markers. Vitamin D status can only be determined by measuring serum 25hydroxyvitamin D levels. Vitamin D3 intake for adults should be 1000 IU per day. The optimal blood level of 25-hydroxyvitamin D for healthy people is 30 to $60 \mathrm{ng} / \mathrm{mL}$. When 25 hydroxyvitamin D levels exceed $150 \mathrm{ng} / \mathrm{mL}$, vitamin $\mathrm{D}$ intoxication ensues. Vitamin D insufficiency causes osteopenia and osteoporosis to develop and worsen, as well as a mineralization problem that leads to osteomalacia. Both are fatsoluble and are absorbed into chylomicrons when consumed. Vitamin D is absorbed via the skin and ingested into the liver, where it is converted to 25hydroxyvitamin $\mathrm{D}$, the primary circulating form of vitamin $\mathrm{D}$ that is used to assess vitamin $\mathrm{D}$ status. However, it is physiologically inactive and requires hydroxylation in the kidney to convert to the biologically active 1, 25-dihydroxy vitamin D. Because 1,252D circulates at 1000 times lower concentrations than $25 \mathrm{D}$ and has a half-life of only 4 to 6 hours compared to 2 weeks for $25 \mathrm{D}$, measuring it is pointless. When a patient is low in vitamin $\mathrm{D}$, circulating PTH levels rise, boosting kidney $1,252 \mathrm{D}$ production. A monthly dose of $100,000 \mathrm{IU}$ of vitamin D2 is similar to $30,000 \mathrm{IU}$ of vitamin D3. Vitamin D deficiency is very common in those over 65 and in people who have osteoporosis. Because vitamin D is only found in a few foods, most people have relied on the sun to meet their vitamin D needs. As a result, anything that inhibits vitamin D production through the skin raises the risk of vitamin D deficiency. Vitamin D insufficiency is caused by intestinal fat malabsorption disorders, which reduce the efficiency of vitamin D absorption. Because vitamin $\mathrm{D}$ is irreversibly sequestered in body fat, obesity is linked to vitamin D insufficiency. Vitamin D3 deficiency or insufficiency is known to cause rickets, osteomalacia, and osteoporosis. It was discovered recently that bone stress fractures are linked to low calcidiol serum levels in young men, even in those who do not have substantial osteoporosis. Vitamin D3 metabolites are referred to as neurosteroids, and they govern systems including behavioural function. As excess of CYP24 might result in vitamin D resistance, it has been suggested that it would be carcinogenic. In the latest investigation, we established definitive proof for the hormonal activity of calcidiol in vitro using a CYP27B1 mutant kidney cell line without la-hydroxylase activity. We validated the hormonal activity of calcidiol in different cell lines employing inhibitors of vitamin D metabolic enzymes. The danger of prostate cancer was inversely associated with calcidiol levels, as measured by quartiles of calcidiol concentrations. When comparing males with calcidiol values beneath $40 \mathrm{nmol} / \mathrm{L}$ to men with calcidiol values above $40 \mathrm{nmol} / \mathrm{L}$, the adjusted relative risk was 1.7. Younger males with low calcidiol levels had the highest risk of prostate cancer. Men with low calcidiol values had a 1.8 -year shorter mean age at diagnosis, and their cancer was more virulent. Vitamin $D$ is generated from 7dehydrocholesterol, a cholesterol precursor found in the skin. When UV-B radiation reaches the skin, it converts the precursor to previtamin D3, which 
then undertakes a thermally induced change into vitamin D3. 25D is a prohormone that acts as a direct precursor of 1, 25-dihydroxy vitamin D 1 , 25 , the active form of vitamin $\mathrm{D}$. The generation of 1,252D, a high-affinity ligand for the vitamin D receptor, is controlled by a single enzyme, 25D-1a-hydroxylase. In research of non-diabetics, it was discovered that insulin sensitivity is inversely related to vitamin $\mathrm{D}$ status and that at $25 \mathrm{D}$ levels of around $46 \mathrm{ng} / \mathrm{mL}$, there is no further reduction in serum glucose. Between the ages of 20 and 80 , the level of 7-dehydrocholesterol in the skin declines by around $50 \%$, lowering the amount of vitamin D3 that older people can produce. It is generally known that people with darker skin produce less vitamin D3 than people with lighter skin, which explains the high incidence of vitamin D insufficiency among darker-skinned people. Heaney measured the 25D responses to different vitamin D3 dosages. The Institute of Medicine National Academy of Sciences recently increased the tolerated maximum consumption threshold of vitamin D from 2000 to $4000 \mathrm{IU} /$ day. Vitamin D toxicity is a clinical condition characterised by both hypervitaminosis D and hypercalcemia. In vitamin D supplementation levels less than 10,000 IU/d, no incidences of vitamin D toxicity have been reported. Vitamin D supplementation has also been shown in recent trials to reduce the incidence of slips, which are a common cause of osteoporotic fractures.

Vitamin D, also appears to reduce the incidence of cancer, according to a substantial amount of data. Even though some research revealed no effect, none of the investigations identified a link between vitamin $\mathrm{D}$ and cancer. The 4-year populationbased randomised, placebo-controlled experiment of Lappe et al is the result of decades of vitamin D research. The autocrine method of activity of vitamin $\mathrm{D}$ is responsible for its anti-cancer effects. The impact of vitamin D in the prevention of numerous autoimmune illnesses is backed up by a large amount of epidemiological evidence. Vitamin D deficiency appears to minimize the chances of multiple sclerosis. It has been demonstrated that microbial agents such as Mycobacterium TB activate the TLR pathway in human monocyte-macrophages, resulting in increased expression of the CYP 27B 25 and vitamin $\mathrm{D}$ receptor genes in those cells. Vitamin D's roles are not restricted to its significant skeletal effects but are also intimately linked to nonskeletal effects. The number of papers related to «Vitamin D» in the PubMed database has risen over time. BICOMB was used to create a MeSH terms-source article matrix, with the source document set being the row and the highfrequency being the column. The term «vitamin D» was searched in the PubMed database, and 4625 documents were identified to have been published between 2015 and 2018. From 4625 articles, BICOMB software was used to derive a total of $11961 \mathrm{MeSH}$ terms with a cumulative frequency of 76125 times. In the winter, mainly in the northern states, respiratory tract infections are more widespread than in the summer. This also applies to the pandemic-like spread of the infectious Coronavirus illness 2019 over the world during the winter months, because the virus is more easily spread at low temperatures. This raises the notion that a lack of vitamin D3 may play a role in the onset and progression of COVID-19. Vitamin D3 deficiency affects approximately one billion individuals worldwide. Vitamin D may play a key role in cell proliferation and immune modulation, influencing many immunological pathways while also improving the protective characteristics of the body's mucous membranes and reducing excessive inflammation. Vitamin D3 alters the synthesis of pro-inflammatory cytokines, which influences the occurrence and severity of viral infections. There is credible evidence that vitamin D3 can suppress the transcription triggered by tumour necrosis factor in human immunodeficiency virus-infected cells that are latently infected. Acute respiratory distress syndrome caused by unopposed production of proinflammatory cytokines IL-6 and TNF- causes an increase in mortality in patients having COVID19. According to a recent meta-analysis, a daily or weekly vitamin D3 intake of 20 to $50 \mathrm{~g}$ led to a considerable decrease of RTIs. When vitamin D3 concentrations approach their ideal range of 40 to $60 \mathrm{ng} / \mathrm{ml}$, the degree of protection is shown to rise. Vitamin D3 inhibits renin and Ang 2 expression, resulting in a considerable reduction in lung 
damage. Vitamin D3 has been linked to increased ACE2/Ang 1-7 activity. As demonstrated in COVID-19, SARS-CoV infection causes ACE2 expression to be down regulated, which leads to acute lung injury and RAS dysregulation, which results in higher inflammation and vascular permeability. Vitamin D is a series of fat-soluble prohormones discovered after the antirachitic activity of cod liver oil was discovered in the early twentieth century. Previtamin D3 is rapidly transformed to vitamin $\mathrm{D}$ in a heat-dependent mechanism. Since vitamin $D$ is a fat-soluble vitamin, it must be absorbed in the presence of dietary fat in the stomach. Vitamin D production is aided by UVB exposure. The connection between vitamin D deficiency and osteoporosis, particularly in the elderly, has been well developed. Additional research is needed to see if 400 IU vitamin D would be enough to prevent fractures in healthy elderly people with normal BMD. Vitamin D deficiency has been identified in patients with vague muscle weakness, aches, and pains. Greater vitamin D levels have been shown to enhance muscle performance and so lessen the risk of falling. In recent times, there has been increasing evidence that vitamin $D$ has a significant relationship with blood pressure. Vitamin D supplementation has been linked to lower blood pressure in humans. One possibility is that vitamin D levels rise as a result of sunlight's protective action. Vitamin D deficiency is also common among MS patients, according to research. Malabsorption is caused by several circumstances, resulting in vitamin D insufficiency. Patients with cystic fibrosis have poor vitamin $\mathrm{D}$ absorption due to pancreatic exocrine insufficiency. Vitamin D insufficiency is common in Crohn's disease patients. As vitamin D is produced in the skin by solar UVB radiation, vitamin $D$ deficiency may increase the risk of colon cancer death. Surprisingly, supplemented vitamin $\mathrm{D}$ had a greater impact on the development of RA than dietary vitamin D. Vitamin D insufficiency has been linked to reduced glucose tolerance since the 1980 s when it was discovered that it suppresses pancreatic production and insulin turnover. Low UVB irradiance, which indicates a low amount of vitamin D, was linked to a higher incidence of type 1 diabetes, whereas the overall prevalence was near zero in areas with high UVB irradiance. Low vitamin $\mathrm{D}$ status caused by a vegetarian diet has recently been discovered to be an independent predictor for active tuberculosis among South Asians. Consumption of vitamin D and calcium drastically reduced tooth loss in older persons, according to a three-year trial. One reason is the fluctuation in vitamin D3 levels during the winter. One of medicine's greatest successes must be the discovery of vitamin $\mathrm{D}$ and the removal of rickets as a serious medical condition. established the structure of vitamin D2 in 1931, and Windaus et al. discovered the structure of vitamin D3 using synthetic methods. Vitamin $\mathrm{D}$ is generally synthesised in the skin by a vigorous photolytic process that produces previtamin $\mathrm{D}$ from a cholesterol derivative, which is then gradually isomerized to vitamin D3. Vitamin D is almost non-existent in the food supply. Another crucial aspect to remember is that vitamin D is essential throughout one's life. Vitamin D activating enzymes have been found. The way vitamin D and its hormone derivatives are disposed of are crucial to metabolism. Through stimulation of the 24hydroxylase, pulses of the vitamin D hormone programme its own demise. The enzyme 24hydroxylase and its control are crucial in determining the circulation levels of the hormonal form of vitamin $\mathrm{D}$. The vitamin $\mathrm{D}$ hormone works in three different ways to raise calcium levels in the blood. The vitamin D endocrine system favours dietary calcium to maintain blood calcium levels under normal circumstances, but when this fails, the mechanism mediates calcium mobilisation from bone and reabsorption in the kidney to meet the organism's requirements. Suda et al, who established that the vitamin D hormone plays a significant role in the terminal differentiation of promyelocytes to monocytes, which are progenitors to gigantic osteoclasts, achieved an amazing development. Vitamin D shortage affects the immune system, particularly $\mathrm{T}$ cell-mediated immunity, whereas too much vitamin $\mathrm{D}$ decreases specific immunological functions. Large dosages of the vitamin D hormone have been shown to inhibit type 1 diabetes mellitus, inhibiting the death of islet cells. 
Vitamin D insufficiency affects an estimated 1 billion people globally, of all races and ages. This outbreak of hypovitaminosis D is largely due to environmental and genetic factors that limit sunshine contact, which is necessary for UVBinduced vitamin D synthesis in the skin. Vitamin D may help prevent cancer, heart disease, fractures and falls, autoimmune illnesses, influenza, type 2 diabetes, and depression, according to new studies. Vitamin D is unusual in that it can be produced in the skin as a result of sun exposure. Only 10-15 percent of dietary calcium and roughly $60 \%$ of phosphorus are digested without vitamin D. Vitamin D supplementation increases calcium and phosphorus absorption by $30-40 \%$ and $80 \%$, respectively. Human milk's vitamin D quantity is linked to the mother's vitamin D status, thus women who replenish with large amounts of vitamin D may have higher vitamin D levels in their milk. Due to their age, older persons are at a higher risk of acquiring vitamin D deficiency. Homebound people, women wearing long robes and head coverings for religious purposes, and those who work in jobs that limit their exposure to sunshine are unlikely to get enough vitamin D from the sun. Darker skin is caused by higher levels of the pigment melanin in the epidermal layer, which reduces the skin's capacity to produce vitamin $\mathrm{D}$ from sunshine. Vitamin D medications may be required by people who have a reduced ability to absorb dietary fat. BMI, waist circumference, and body fat are all inversely related to $25 \mathrm{D}$ levels, although age, lean body mass, and vitamin D intake are all positively related. In a three-year study of nondiabetic people aged 65 and over, those who got 700 IU of vitamin $\mathrm{D}$ had a lower increase in fasting plasma glucose than those who received a placebo. Low vitamin D levels have been linked to significant cognitive deterioration in the elderly. It has been postulated that chronically low vitamin D levels may play a crucial role in Parkinson's disease aetiology. VDD may be the seasonal stimulation that causes influenza outbreaks during the winter months. VDD was linked to a roughly three-fold increase in the risk of bacterial vaginosis. Fibromyalgia is sometimes misinterpreted as vitamin D insufficiency. Orange juice enhanced with vitamin
D is a new healthy source of vitamin D. Velluz et al. were the first to find previtamin D3 and demonstrate its conversion to vitamin D3. As the number of UVB photons absorbed by 7dehydrocholesterol determines the quantity of previtamin D3 produced in the skin, any method that reduces the number of UVB photons accessing the epidermis or reduces the amount of 7dehydrocholesterol in the skin will result in significant reductions in or total removal of vitamin D3 production in the skin. All solar light beneath $290 \mathrm{~nm}$ is efficiently absorbed by the stratospheric ozone layer. However, UVB light over $290 \mathrm{~nm}$, which is responsible for the production of previtamin D3 in the skin, can be absorbed by the ozone layer. The UV radiation that 7-dehydrocholesterol may capture has energy as low as $315 \mathrm{~nm}$. Many people get enough vitamin $\mathrm{D}$ from regular sun exposure to meet their body's requirements. Vitamin D3 is created in sufficient quantities in the skin during the spring, summer, and fall to be retained in body fat, where it can be mobilised during the winter months when little, if any, vitamin D3 is generated in the skin. The small intestine absorbs just $10-15$ percent of dietary calcium lacking vitamin $\mathrm{D}$. Adults with vitamin D deficiency develop secondary hyperparathyroidism, which can worsen and accelerate osteoporosis. Vitamin D deficiency is a danger for the elderly due to inadequate dietary vitamin D consumption and reduced sun exposure. The relevance of this hormone in the total treatment and prevention of chronic diseases is at the core of the study, as the number of persons with VDD continues to rise. Patients who are vitamin D deficient should receive treatment with either vitamin D2 or vitamin D3. Further research is needed before recommending screening or prescribing vitamin $\mathrm{D}$ to achieve the non-calcemic effect for cardiovascular protection in people who aren't at risk of deficiency.

\section{Conclusion:}

This research review's purpose is to help the reader understand different aspects posed by the research on the topic of Therapeutic Combination of Multivitamin. This is significant because it gives insights about the different pharmacological 
effects of different Vitamin groups like Vitamin A, Vitamin B family, Vitamin C and Vitamin D. There has been much research and discussion conducted on these opinions of them and the therapeutic applications of Multivitamins. Most of the research found was on the details of using different Vitamin groups along with other medications to treat a variety of disorders. More research and testing is required to gain a better understanding of the pharmacological effects of the Vitamin groups.

\section{Acknowledgement:}

We would like to thank our supervisor/guide Bharat Kwatra, from Invenzion Labs Inc and our Sub-mentor Chelsea Rumao. whose expertise were invaluable in formulating the research questions, methodology and drawing conclusions. Their insightful feedback and guidance pushed us to sharpen our thinking and brought our work to a higher level.

\section{Ethics Approval and Consent to Participate.}

Not applicable.

\section{Human and Animal Rights}

No Animals/Humans were used for studies that are the basis of this research.

\section{Consent for Publication}

Not applicable.

\section{Availability of Data and Materials}

The author confirms that the data supporting the findings of this research are available within the article.

\section{Funding Acknowledgement and Conflict of Interest}

The authors whose names are listed immediately above certify that they have NO affiliations with or involvement in any organization or entity with any financial interest (such as honoraria; educational grants; participation in speakers' bureaus; membership, employment, consultancies, stock ownership, or other equity interest; and expert testimony or patent-licensing arrangements), or non-financial interest (such as personal or professional relationships, affiliations, knowledge or beliefs) in the subject matter or materials discussed in this manuscript.

\section{References:}

1. Boulkrane, M. S. et al. COVID-19 Disease and Vitamin D: A Mini-Review. Frontiers in Pharmacology vol. 11 (2020).

2. Yang, A. et al. Identification of recent trends in research on Vitamin D: A quantitative and co-word analysis. Med. Sci. Monit. 25, (2019).

3. Nair, R. \& Maseeh, A. Vitamin D: The sunshine vitamin. Journal of Pharmacology and Pharmacotherapeutics vol. 3 (2012).

4. Lappe, J. M. The role of vitamin D in human health: A paradigm shift. Complementary Health Practice Review vol. 16 (2011).

5. Zhang, R. \& Naughton, D. P. Vitamin D in health and disease: Current perspectives. Nutrition Journal vol. 9 (2010).

6. Tuohimaa, P. Vitamin D, aging, and cancer. in Nutrition Reviews vol. 66 (2008).

7. Holick, M. F. The role of vitamin D for bone health and fracture prevention. Current Osteoporosis Reports vol. 4 (2006).

8. Wolpowitz, D. \& Gilchrest, B. A. The vitamin D questions: How much do you need and how should you get it? Journal of the American Academy of Dermatology vol. 54 (2006).

9. DeLuca, H. F. Overview of general physiologic features and functions of vitamin D. The American journal of clinical nutrition vol. 80 (2004).

10. Holick, M. F. Vitamin D: Importance in the prevention of cancers, type 1 diabetes, heart disease, and osteoporosis. in American Journal of Clinical Nutrition vol. 79 (2004).

11. Fiorillo, M., Tóth, F., Sotgia, F. \& Lisanti, M. P. Doxycycline, Azithromycin and vitamin C (DAV): A potent combination therapy for targeting mitochondria and eradicating cancer stem cells (CSCs). Aging (Albany. NY). 11, (2019).

12. Lenz, T. L. Effects of Food on the Most Commonly Used Medications in School-Age Children. American Journal of Lifestyle Medicine vol. 3 (2009). 
13. Alcantara-Martos, T., Delgado-Martinez, A. D., Vega, M. V., Carrascal, M. T. \& Munuera-Martinez, L. Effect of vitamin $\mathrm{C}$ on fracture healing in elderly Osteogenic Disorder Shionogi rats. J. Bone Jt. Surg. - Ser. B 89, (2007).

14. Cursino, L., Chartone-Souza, E. \& Nascimento, A. M. A. Synergic interaction between ascorbic acid and antibiotics against Pseudomonas aeruginosa. Brazilian Arch. Biol. Technol. 48, (2005).

15. Scheid, D. C. \& Hamm, R. M. Acute bacterial rhinosinusitis in adults: Part II. Treatment. American Family Physician vol. 70 (2004).

16. Gordon, J. E. \& Schooff, M. Can high-dose supplementation with vitamins $\mathrm{C}$ and $\mathrm{E}$, beta carotene, and zinc slow the progression of macular degeneration? Journal of Family Practice vol. 51 (2002).

17. Johnston, C. Physicians should warn patients about nutrition-related scams, doctor advises. $C M A J$ vol. 147 (1992).

18. Alsayari, A., Muhsinah, A. Bin, Almaghaslah, D., Annadurai, S. \& Wahab, S. Pharmacological efficacy of ginseng against respiratory tract infections. Molecules vol. 26 (2021).

19. Loukou, I. et al. Association of vitamin A status with lung function in children and adolescents with cystic fibrosis. Pediatr. Investig. 5, (2021).

20. Fan, S. et al. Panax ginseng clinical trials: Current status and future perspectives. Biomedicine and Pharmacotherapy vol. 132 (2020).

21. Coon, J. T. \& Ernst, E. Panax ginseng: A systematic review of adverse effects and drug interactions. Drug Safety vol. 25 (2002).

22. Bendich, A. \& Langseth, L. Safety of vitamin A. American Journal of Clinical Nutrition vol. 49 (1989).
23. Warwick, W. J., Hansen, L. G. \& Sharp, H. Absorption of Vitamin A in Patients with Cystic Fibrosis: Absorption Is Best with Emulsified Vitamin A Alcohol. Clin. Pediatr. (Phila). 15, (1976).

24. Mock, D. M. Biotin: From nutrition to therapeutics. J. Nutr. 147, (2017).

25. Rolfe, H. M. A review of nicotinamide: Treatment of skin diseases and potential side effects. Journal of Cosmetic Dermatology vol. 13 (2014).

26. Wohlrab, J. \& Kreft, D. Niacinamidemechanisms of action and its topical use in dermatology. Skin Pharmacology and Physiology vol. 27 (2014).

27. Sriram, K., Manzanares, W. \& Joseph, K. Thiamine in nutrition therapy. Nutrition in Clinical Practice vol. 27 (2012).

28. Horváth, Z. \& Vécsei, L. Treatment with pantethine. J. Evidence-Based Complement. Altern. Med. 16, (2011).

29. Gisondi, P., Fantuzzi, F., Malerba, M. \& Girolomoni, G. Folic acid in general medicine and dermatology. Journal of Dermatological Treatment vol. 18 (2007).

30. Ryan-Harshman, M. \& Aldoori, W. Vitamin B12 and health. Canadian Family Physician vol. 54 (2008).

31. Toyosawa, T., Suzuki, M., Kodama, K. \& Araki, S. Potentiation by amino acid of the therapeutic effect of highly purified vitamin B2 in mice with lipopolysaccharide-induced shock. Eur. J. Pharmacol. 493, (2004).

32. Komatsu, S., Yanaka, N., Matsubara, K. \& Kato, N. Antitumor effect of vitamin B6 and its mechanisms. in Biochimica et Biophysica Acta - Proteins and Proteomics vol. 1647 (2003). 